\title{
Advances In Research Of Vehicular Ad-hoc Network
}

\author{
Tong Liu ${ }^{1, a}$, Defu Cheng ${ }^{2, c}$ and Yue Huang ${ }^{3, d}$ \\ ${ }^{1}$ College of Instrumentation \& Electrical Engineering / Center for Computer Fundamental Education, \\ Jilin University, Changchun, China \\ ${ }^{2}$ College of Instrumentation \& Electrical Engineering, Jilin University, Changchun, China \\ ${ }^{3}$ College of Computer Science and Technology/ Center for Computer Fundamental Education, Jilin \\ University, Changchun, China

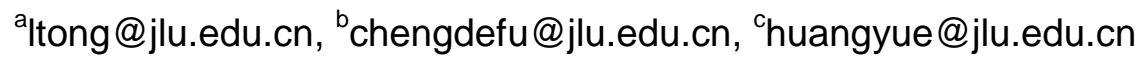

Keywords: VANET; protocol; routing; broadcast; quality of service; security; simulation

\begin{abstract}
Vehicular Ad-Hoc Network (VANET) is extremely potent in improving the security and efficiency of vehicles and roads, as well as the comfort of drivers and passengers. The standardization and development of VANET have received attention from various fields. Recent studies focus on these fields such as routing protocols, broadcast, quality of service, security, and simulation. In this paper, we review the new advances in these fields and elaborate the new achievements and newly introduced techniques and methods. Based on this, we summarize the challenges encountered in research of VANET.
\end{abstract}

\section{Introduction}

Vehicular Ad-Hoc Network (VANET) aims to build a mobile network using the running vehicles as nodes. In VANET, each involved vehicle is treated as a wire-less router or node, and the vehicles within a distance of $300-1000 \mathrm{~m}$ are interconnected, thereby creating a widespread network. The development of VANET will inspire a growing number of wireless communication applications, including vehicle security, automated payment, traffic management, enhanced navigation, and position-based services [1] .

\section{Advances in Research of Vanet}

\section{Routing protocol of VANET}

It necessitates a reliable routing algorithm that meets the demands of VANET[2]. Many efforts have been devoted to this field. For instance, a priority-based safe distributed routing protocol is based on broadcast communication and does not require routing maintenance [3]. The specific message priority and the one-hop maximum transmission distance correspond to different QoS, and thus the competition mechanism is used in secure communication and election [4]. A road and traffic perceptive routing protocol (RTRP) uses distance and density to compute the optimal route for transmitting data packets [4]. A new protocol was introduced into the connection between VANET and Internet, and the vehicle moving rules are used to predict a vehicle's future behaviors and to select the longest-lasting route and connect it to the wired network [5]. For both sparse and dense networks, a minimum delay geographic routing protocol connected with perception can adapt to the changeable network status; this protocol was tested in special vehicle environments, such as when the target vehicle is far away from the expected position, or when the excepted next-hop node is not discovered [6].

Currently, the greed routing selection based on position information has been the most widely used designing thought. In this type of protocols, a node does not search any routing before sending data, without needing other topological information, and the whole data transmission does not require the building or maintenance of routing. A mobile node directly formulates a data transmission strategy according to the position information of itself, the neighbor nodes, and the target node. The common 
position-based routing protocols include LAR, GPSR, LOTAR, Terminodes Routing, and Gird Routing.

In VANET, since GPS can easily be installed in vehicles, this type of protocols is feasible. For this type, the routing protocol based on the greedy algorithm is the most mature.

Based on position information, the vehicle's in-built functions such as navigation and electronic maps (E-maps) can be utilized to develop a routing mechanism based on road topological information. In this type of protocols, before sending data, a node first searches a routing and it saves the routing table after success. However, this routing does not point to the intermediate node sequence, but a data transmission path in space, which is constituted of intersections. The multihop transmission avoids the traversing of roadside obstacles, thus improving the reliability of data transmission. The topology-based routing protocols include AODV, GSR, TORA, DSR, and OLSR.

The topology-based position routing protocols well integrate the position information and road structure, thus improving channel condition and data transmission reliability. However, in VANET, the frequent variation of topological structure will lead to frequent route invalidation and lower data transmission quality. Once a data packet is transmitted to a vehicle-scarce area, it may start a nearby routing if it cannot find a suitable next-hop, thereby resulting in larger delay or even packet loss.

The VANET routing protocols should develop to map-based routing (MBR) protocols. At present, few concrete protocols have been proposed. Utilization of vehicle characteristics (e.g. vehicular GPS and E-maps) will help a node to obtain topological information about itself, neighbor nodes and roads. Using the functions such as route planning in the navigation system, then several finite sets including node set, road set, and steering limitation set can be used to describe and predict the overall topology of the whole network, and to more accurately and fast compute the optimal source-to-target route. For instance, Spatial Aware Routing (SAR) algorithm is developed from GPSR routing protocol, and its main ideas are to construct the network topology using the static digital map data, and then use a graph algorithm to find a source-to-target route. According to the discovered route, the data packet will be transmitted via the source routing to the target node. With use of both geographic stateless routing and node position digital maps, the GeoSVR protocol optimizes the transmission route and solves the problems of local maximum and sparse connection, and then uses a limiting transmission algorithm to overcome the problem of unreliable wire-less channels.

Many routing protocols adopt the concept of divide-and-conquer, which aims to solve the gigantic computation of topological information in the whole network, and thus divides the whole network into several groups, or dividing a route into several sections, so as to compute and manage road information.

\section{Qos of Vanet}

\section{Analysis of QoS of VANET}

The application of VANET includes data business and real-time business.

Data business mainly involves TCP in the transport layer as a basic transmission control protocol, with low requirement for real-time performance; the improvement of QoS is reflected by throughput and fairness. This not only requires deep study into MAC layer protocol, and also in improving the TCP protocol of transport layer. Then we analyze the effects of DCF, FCR and NASD on TCP performance.

In case of large network scale and heavy network load, the loss of frame is high in the MAC layer of DCF, the channel conflict occurs frequently, so that the TCP transmission end will frequently reduce congestion window because of data packet loss, entering the stage of slow start. The FCR mechanism can rapidly eliminate channel conflict, and reduce the probability of TCP data packet loss. However, after a single station successfully occupies a channel, it nearly occupies all channel resources, while other stations will re-occupy channels after a long time, severely affecting the stability of TCP data transmission and rapidly reducing the throughput of TCP. When the MAC layer adopts the NSAD mechanism, it will monitor the real-time network load and dynamically adjust the 
initial competition window. These actions will smooth the transmission of TCP data packet, reduce transmission interval, stably transmit data packets at the maximum throughput and effectively avoid the long-term occupation of channel by a single station.

To improve TCP throughput and maintain stability from the MAC layer, we should efficiently reduce and decompose channel conflict, and prevent a single station from continuous conflicts. It should also avoid TCP frequent return and slow start-up, reduce TCP throughput, and maintain stable TCP throughput. Among three MAC mechanisms, the network throughput is low in DCF and TCP, but highest in FCR; the throughput of TCP is smaller than NSAD, because it is inferior to NSAD in keeping the stable transmission of ACK packages.

Real-time business mainly utilizes EDCA. EDCA mechanism utilizes the priority division algorithm, and the priority of data business is the lowest, and thus the average throughput of data business in DCF mechanism is obviously higher than in EDCA mechanism, but DCF mechanism cannot guarantee the QoS of real-time business. When a node is moving at relatively static or low velocity, the average delay for real-time business in EDCA mechanism is small, and the delay jitter is reduced to some extent, but when the network load is large, the average delay jitter will be greatly improved. When the node's relative movement speed is increasing, the average delay and delay jitter will both be increased slowly in EDCA mechanism but suddenly in DCF mechanism. However, when the network load is large or the relative movement is fast, the delay and delay jitter of real-time business are still large, not ensuring the QoS of real-time business. Therefore, many efforts have been devoted to improving the channel access control mechanism of standard EDCA. The basic ideas are to dynamically adjust the size of $\mathrm{CW}$ and to estimate network flow using the probability of collision, based on the flow status of network. For instance, AEDCF, EDCF-DM and ACT-EDCF mechanisms are mainly based on the close relationship between the size of $\mathrm{CW}$ and the network capacity.

\section{QoS routing}

The QoS routing in Ad Hoc network is mainly responsible for finding, building and maintaining the system of feasible paths, supporting the management control mechanisms (e.g. accepting the call), and finally ensuring the effective utilization of whole-network resources. VANET does not have many QoS routing protocols, and research is continuing, and the latest hot QoS routing protocols are listed below.

Based on node movement conditions and the changing laws of traffic lanterns, GV Grid [7] selected the grids as the optimal path where the nodes are moving at similar speeds. This is equal to finding a long-standing routing. The expected survival time of a routing is decided by the moving velocity and direction of nodes and the basic characteristics of roads. However, along with the movement of nodes, a new optimal path may appear between the source and target nodes, but during the routing repair process, the protocol will not re-compute the optimal path, but instead regard the first-time computed path as always the optimal path from the source to target nodes. Multi-Hop Routing protocol for Urban VANET (MURU) [8] uses the routing survival time as an index, selects the most stable channel as the routing, thereby ensuring the QoS performance indices of Vanet, such as delay and packet loss rate. The relative velocity and direction of the nodes are used to estimate the possibility of routing rupture within a given period, and the MURU protocol aims to find the routing with the lowest cumulative possibility. Delay and Reliability Constrained QoS'routing algorithm (DeReQ) considers two factors of latency and link stability, based on the improvement in AODV and LBM of routing protocol, aiming to find a QoS routing between the source to the target nodes, which contains relatively stable links and meets delay requirement.

\section{Broadcast}

\section{VANET-based broadcast compression}

The existing VANET broadcast protocols are mostly extended from traditional MANET broadcast protocols. Since the VANET nodes cannot detect conflict and are highly mobile, we cannot understand the topological information of the whole network before broadcasting, and thus the 
VANET broadcast utilizes a simple flooding algorithm, but the flooding broadcast will generate redundancy, competition and conflict. In response, there are now numerous broadcast suppression algorithms, and the major algorithms are listed below.

The position-based algorithms: with the help of GPS, the unit vehicles can obtain information about its own position and velocity, and thus the position-based broadcast techniques are more advantageous in VANET. According to the position of the sender of the repeated broadcast, from which a node receives the broadcast, it computes the area the re-broadcast can cover, if this area is smaller than the limit A, it cancels re-broadcast. Most VANET broadcast protocols perform optimization using the positional information of vehicles, such as DDT, ODAM, SNB, S1-PB, EDB, and RBRS.

Algorithms based on information of neighbor nodes: based on local information, it constructs the smallest connected set, and any node in the network is either in this connected set or near a node in this connected set. After a node receives the broadcast, it decides to re-broadcast according to the coverage conditions. Si-Ho Cha et al. proposed a clustering algorithm based on a connected domination set, and this set can be used to construct a fictitious backbone network, thereby improving the extensibility of networks. Chi Trung Ngo and Hoon Oh put forward a gateway discovery algorithm based on connected domination set, and through link duration, they build a connected domination set, in which one transmission by any vehicle can be received by all vehicles. [11-12]

\section{Performance indices for broadcast protocols based on IEEE 802.11}

They design efficient message broadcast protocols targeted at traffic information applications, the performance of IEEE802.11 broadcast protocols in VANET network environment, and the key factors affecting such performance. According to the driving characteristics, they consider on-road vehicles distribution, and within certain range, the vehicles can be regarded as average distribution; suppose density is $\lambda$, node transmission range is $r$, then the number of covered nodes is $n=2 \lambda r$. With the ideal channel as example, suppose the arrival of broadcast package in the MAC queue obeys Possion distribution with mean of $\mu$, and the queuing rule is "first come first served", satisfying M/G/1 queue discipline. The transmission probability of each node is related to the size of competition window, and the number of nodes in the transmission range of this broadcast node [13]. Based on Markov chains, they construct the service delay and network throughput of this queue, and then:

$$
\tau=\frac{2(1-p)}{W+1-2 p}
$$

At each time slot, $\mathrm{W}$ is the probability of transmitting broadcast message by this node, $\mathrm{W}$ is the status probability matrix and $\mathrm{P}$ is the status shift probability matrix. Then at slot $\sigma$, within the transmission range $\mathrm{r}$ of this node, the probability of transmitting broadcast information by a node can be expressed as:

$$
p_{b}=1-(1-\tau)^{n}
$$

Then the probability of a node successfully sending the broadcast message can be expressed as:

$$
p_{s}=\frac{n \tau(1-\tau)^{n-1}}{p_{b}}
$$

The time of broadcast packages with average load length $\mathrm{E}$ can be expressed as $\mathrm{T}$, then

$$
T=T_{M A C+P H Y}+\frac{E}{b}+D I F S+\delta
$$

$T_{M A C+P H Y}$ denotes the package head of broadcast package, and $\delta$ is the transmission delay. For this node, the chain time occupied by the successfully transmitted broadcast information is equal to the time occupied by the occurrence of collision, then $\mathrm{T}=\mathrm{Ts}=\mathrm{Tc}$.

The mean of virtual slots is [14] :

$$
T_{V}=\left(1-p_{b}\right) \sigma+p_{b} p_{s} T_{s}+p_{b}\left(1-p_{s}\right) T_{c}
$$

The normalized network throughput can be expressed as: 
$S=\frac{p_{b} p_{s} \times\left(S I Z E_{\text {packet }} / \text { Rate }\right)}{\left(1-p_{b}\right) \sigma+p_{b} T_{s}}$

The broadcast average delay means the average time needed to successfully transmit the first broadcast package, then:

$\bar{E}=E_{\text {bach }} T_{V}$

$\bar{E}$ is the product from the number of virtual time slots and the virtual slot, and is the number of shirking slot before the successful transmission. Since the probability of picking this node is equally divided within $[0, \mathrm{~W}]$, then $E_{\text {back }}=\frac{W+1}{2}$.

\section{Security}

\section{Message validation}

Vehicles can share information, such as road and traffic conditions, and adopt multiple digital signatures. There are generally two methods of signing: joint signature and onion signature. In joint signature, the signature and certificate of each intermediate node are directly attached to the message, and there are $\mathrm{n}$ signatures. In onion signature, the message is hached into a fixed-length string, and then each signature is resigned. During message transmission, the legality will be also transmitted and validated.

VANET requires frequent independent identity validation, and the digital signature should authenticate abundant data, and the "public key infrastructure" (PKI) is used in authentication [20]. Before transmission of secure messages, the vehicles sign private keys, including CA certificate. Tamper-proof devices should be installed on vehicles to store secret messages and send signed keys.

At present, IEEE1609.2 provides VANET with security services, and thus elliptic curve digital signature algorithm (ECDSA) is used to validate messages and certificate [21]. The validation program may buffer the signer's certificate and public key, necessitating economic use of wire-less bandwith. All manufacturers are devoted to solving VANET security and privacy.

\section{Ad Hoc secure strategy}

Ad Hoc network is independent of any fixed infrastructure, without central node, and the traditional network authentication mechanism is unfeasible. Generally, distributed authentication is used to achieve identity faith in the nodes of Ad Hoc network, namely the trust policy of the trusted third party.

Self-safety certificate trust model, in which a security strategy with trust dispersion is introduced into the Ad Hoc network: the trust to the traditional certificate authorization institute CA is dispersed into joint trust of several nodes. The core technology is the sharing of threshold secret. $n$ key management servers share the certificate signing ability, $(n, t+1)$ threshold code design is used to divide key $\mathrm{k}$ into $\mathrm{n}$ portions $(\mathrm{s} 1, \mathrm{~s} 2, \ldots, \mathrm{sn})$, give each server with one portion to form partial signing, then are assembled by a combiner to at least $\mathrm{t}+\mathrm{l}$ correct partial signatures, synthesized into a correct signature. Less than $t+1$ participators cannot reconstruct signature $S$. The most widely used is Shamirnt threshold scheme, in which, when the original sharers' secret shares are unchanged, new sharers can be added. The implementation of classification scheme will give each sharer with different numbers of secret shares. The complexity is calculated by secret recovery algorithm to be $\mathrm{O}(\mathrm{n} 3)$.

The self-issue certificate trust model has no unified CA, but each node in Ad Hoc network issues itself a secret key and certificate. With a trustful way in processing, each user stores and distributes a certificate, and maintains a local certificate warehouse. When two users expect to authenticate the other party's public key, they combine their local certificate warehouses and find a certificate chain, 
and the trust relationship between nodes is built via this chain. This scheme does not necessitate any specific authentication institution, thus conforming to the characteristics of Ad Hoc network, without a center node, and all nodes are not divided by priority. However, the certificate chain of two communication parties exists with certain probability, and especially at the initial stage of network building, there is time when the two nodes are inaccessible, without definite trust guarantee.

\section{Conclusions}

In the past decade, VANET and relevant fields have been extensively studied worldwide. VANET is highly dynamic. VANET is a multi-hop wire-less network. The routing algorithms in this field are a challenge, and broadcast is a typical application, such as emergency message, traffic information, and notice and advertising messages. However, flooding is still tough. We summarized the hotspots in VANET, including security, routing, quality of service, and broadcasting technology, and discussed the challenges encountered in VANET.

\section{References}

[1] Ping Yi, et al.Wireless ad hoc networks and peer-to-peer networks: Principles and Safety[M]. Tsinghua University press, 2009.

[2] Luo Juan, Lu Zhen, Li Renfa. Network-Coding Based Multicast Routing in VANET. [J]. Journal of Computer Research and Development, 2011, 48(9): 1616-1622.

[3] C. Suthaputchakun, S. Zhili. Priority based Routing Protocol in Vehicular Ad hoc Network[J]. IEEE Symposium on Computers and Communications (ISCC), 2011, Pages 723-728.

[4] Pham Thi Hong, Hyunhee Park, Chul-Hee Kang. A Road and Traffic-aware Routing Protocol in Vehicular Ad hoc Networks[C]. In 13Th International Conference on Advanced Communication Technology (ICACT), 2011.

[5] Abderrahim Benslimane, Saman Barghib Chadi Assi. An efficient routing protocol for connecting vehicular networks to the Internet[J]. Pervasive and Mobile Computing. Volume 7, Issue 1, February 2011.

[6] Kaveh Shafiee, Victor C.M. Leung. Connectivity-aware minimum-delay geographic routing with vehicle tracking in VANETs[J]. Ad Hoc Networks, Volume 9, Issue 2, March 2011, Pages 131-141.

[7] Weihua Sun, Hirozumi, yamaguehi. GVGrid: A QoS Routing Protocol for Vehieular Ad Hoc Networks[J]. In Proc. of the 2006 international conference on Wireless communications and mobile computing,2006:130-139.

[8] Lidstrom.K, Larsson.T. A Spatial QoS Requirements Specification for V2V Applications[C]. IEEE Intelligent Vehicles Symposium,2010:548-553.

[9] Zhang Q Dharma PA. KIM JS. Probabilistic broadcasting based on coverage area and neighbor confirmation in mobile ad hoc networks[C]. In: Proc. of the IEEE Communications Society. New York: IEEE Press, 2004. 96-101.

[10] N.Wisitpongphan, O.K. Tonguz, J.S. Parikh, et al. Broadcast Storm Mitigation Techniques in Vehicular Ad Hoc Networks [J]. IEEE Wireless Communications. Dec. 2007, 14(6): 84-94.

[11] Si-Ho Cha, Min-Woo Ryu, Kyu-Ho Kim, Byoung-Chan Jeon. Applying Connected Dominating Set to Broadcastingin Vehicular Ad Hoc Networks [C]. Information Science and Applications (ICISA), 2013 International Conference on: 24-26 June 2013. 2013:1 - 2

[12] Chi Trung Ngo, Hoon Oh. A Gateway Discovery Approach Using Link Persistence Based Connected Dominating Sets for Vehicular Ad Hoc Networks [J]. Ad-hoc, Mobile, and Wireless Network,Lecture Notes in Computer Science,Volume 7960, 2013:305-316.

[13] Liu HongFei. Study on the Model and Optimization Methods of Information Broadcasting in VANET[D]. Chongqing University,2009.

[14] Tomoya S, Hiroshi I. A Back-off Scheme for IEEE802.11 Wireless LANs[C] . 11th IEEE Singapore International Conference on Communication Systems, ICCS 2008:1115-1119. 